\title{
In-situ growth of crystalline Ge nanowires by using nanotubes as template
}

\author{
Wei-Qiang Han, * Lijun Wu, * Yimei Zhu, * and Myron Strongin** \\ * Center for Functional Nanomaterials, Brookhaven National Laboratory, Upton, NY 11973-5000 \\ ** Department of Physics, Brookhaven National Laboratory, Upton, NY 11973-5000
}

One-dimensional nanomaterials, such as nanotubes and nanowires, have been investigated for potential applications because of their unique electrical, optical and mechanical properties [1-3]. Wu et al. reported the formation of Germanium $(\mathrm{Ge})$ nanowires by in-situ TEM studies. In their work, micrometer-sized Ge particles were dispersed on transmission electron microscope (TEM) grids together with monodispersed Au nanoclusters. This is a vapor-liquid-solid nanowire growth process with $\mathrm{Au}$ as the catalyst. The heating temperature was 800-900 $\mathrm{C}^{\circ}$ [4]. Carbon nanotubes (CNTs) have been used as templates to synthesize filled and coated nanotubes, as well as carbide and nitride nanowires [2, 5-7]. During the formation of nanowires through the CNT-confined reaction, CNTs were used as templates and were fully or partially consumed as carbon oxides. Different from the above work, here we report a novel in-situ TEM route to grow Ge nanowires by using nanotubes as templates, without using a catalyst and without consumption of nanotubes. Crystalline Ge nanowires grow parallel and are bonded to the nanotubes.

In the present work, nanotubes are first dispersed on TEM grids and then put into a high vacuum chamber where they are coated with Ge by a physical vapor deposition technique. In-situ growth of Ge nanowires was then observed using JEOL-3000 F TEM equipped with a Gatan heating stage. The Ge coating is uniformly amorphous, though only one-side is coated because of shadow-effect of the nanotubes (Figure 1). The thickness of the coating is about 2-3 nm. We varied the temperatures to monitor the crystallization of the amorphous Ge coating. When the heating temperature was raised to $350 \mathrm{C}^{\circ}$, crystalline Ge nanoparticels start to form within the amorphous Ge. The size and amount of crystalline Ge nanoparticles increased with increasing of heating temperature. In the end, the sample was heated at $760 \mathrm{C}^{\circ}$ for a half hour and then cooled naturally. After the annealing, we found that amorphous Ge was completely crystallized and some of them are single crystals. More interestingly, the curved coatings stretched to flat nanowires, which are parallel and bonded with the nanotubes. The nanotubes still keep their original structure without any consumption. Figure 2 shows a high resolution TEM image of a single crystalline nanowire parallel and bonded with a nanotube. The diameter of the nanotube is about $9.5 \mathrm{~nm}$ and the width of the Ge nanowire is about $15.9 \mathrm{~nm}$. The axis direction of the Ge nanowire is [111]. We will proposal a model for the growth mechanism of the nanowires.

In summary, we report a simple and efficient route for the formation of novel nanostructures composed of nanowires bonded to a nanotube, and which are expected to have novel physical properties and important applications. This route is not only good for the formation of Genanowire/nanotube parallel nanostructures, but is also good for the formation of other nanowire/nanotube parallel nanostructures.

References

[1] S. Iijima, Nature 354 (1991) 56. 
[2] W. Han et al., Science 277 (1997)1287.

[3] J. Hu et al., Acc. Chem. Res. 32 (1999) 435.

[4] Y. Wu and P. Yang, J. Am. Chem. Soc. 123 (2001) 3165.

[5] W. Han and A. Zettl, Appl. Phys. Lett. 84 (2004) 2644.

[6] P. M. Ajayan and S. Iijima, Nature 361 (1993) 333.

[7] W. Han, A. Zettl., J. Am. Chem. Soc..125 (2003) 2062.

[8] This research was supported by the U. S. DOE under contract DE-AC02-98CH10886.

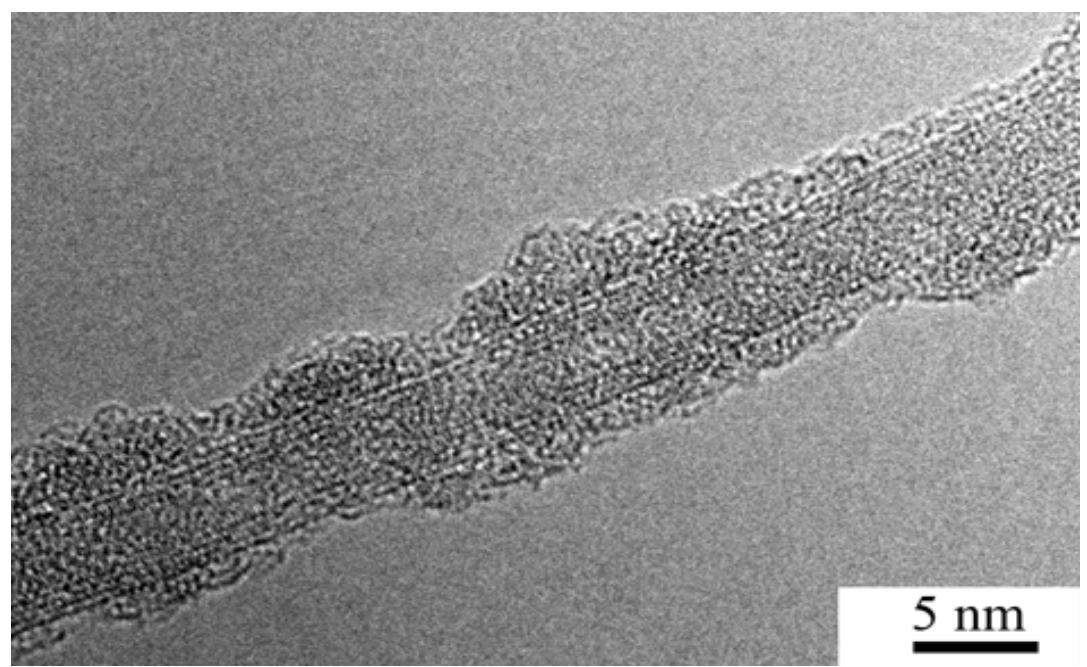

FIG. 1. High-resolution TEM image of a nanotube coated with amorphous Ge.

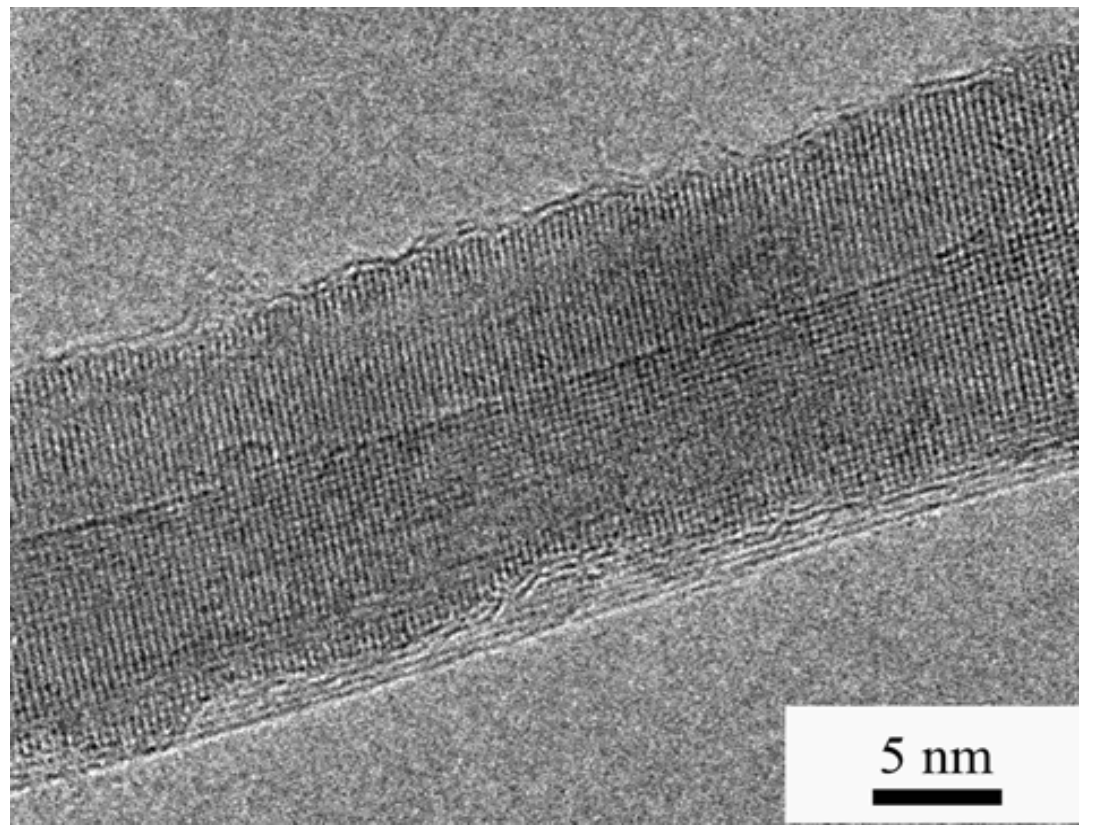

FIG. 2. High-resolution TEM image of a single-crystalline Ge nanowire parallel and bonded with a nanotube, which is the template for the formation of the Ge nanowire. 